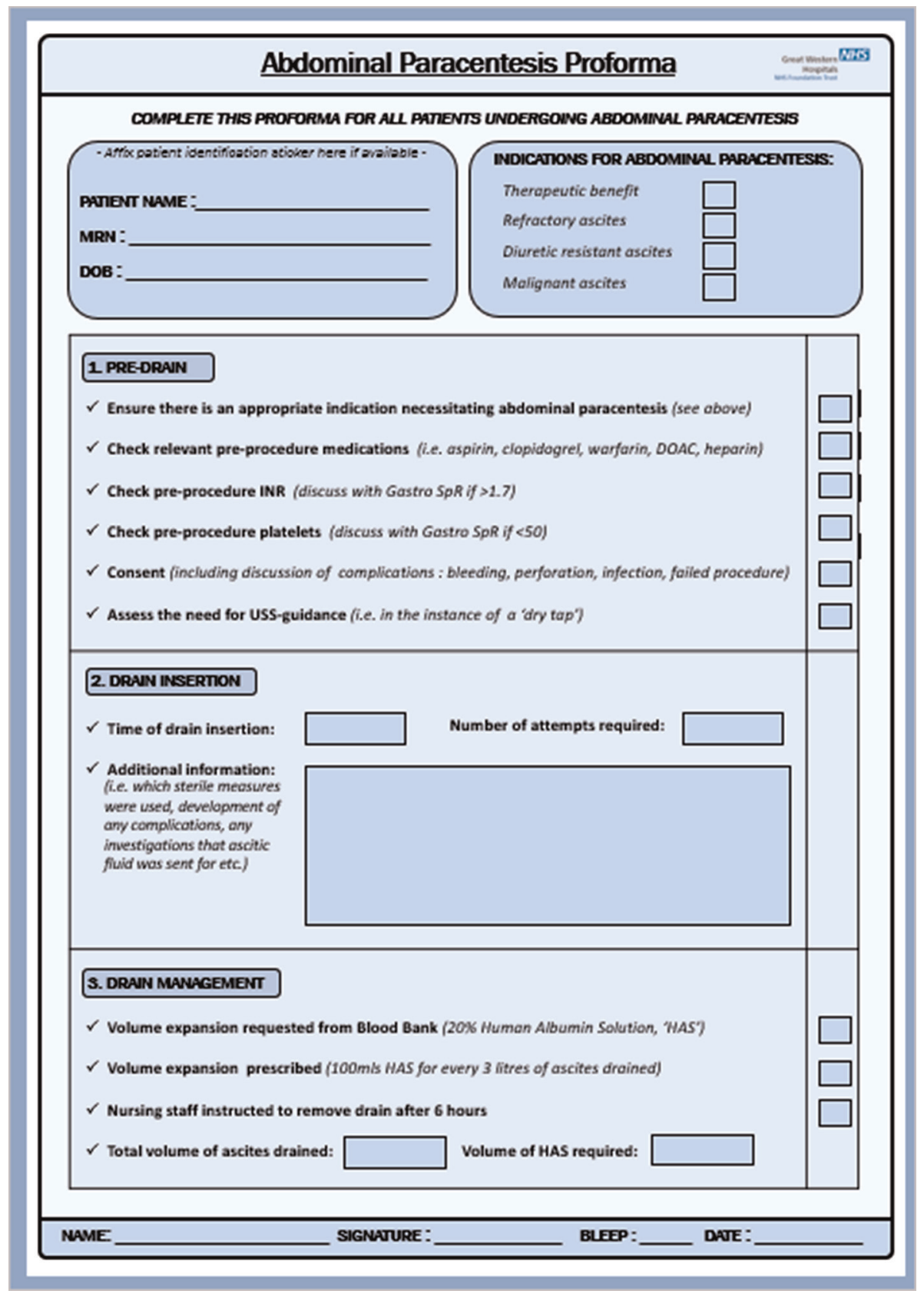

Abstract PWE-106 Figure 1

introduction of a dedicated proforma. There remains further scope to develop this, ideally supported by practical training.

\section{PWE-107 DIRECT OBSERVATION OF PROCEDURAL SKILLS (DOPS) ASSESSMENT IN GASTROSCOPY: VALIDITY AND COMPETENCY DEVELOPMENT DURING TRAINING}

${ }^{1}$ Keith Siau*, ${ }^{2}$ Prof James Crossley, ${ }^{3}$ Paul Dunckley, ${ }^{4}$ Gavin Johnson, ${ }^{5}$ Mark Feeney, ${ }^{6}$ Neil Hawkes, ${ }^{7}$ lan Beales. ${ }^{1}$ oint Advisory Group on Gastrointestinal Endoscopy, London, UK; ${ }^{2}$ University of Sheffield, Sheffield, UK; ${ }^{3}$ Gloucestershire Hospitals NHSFT, Gloucester, UK; ${ }^{4}$ University College London Hospitals NHSFT, London, UK; ${ }^{5}$ Torbay and South Devon NHSFT, Torquay, UK; ${ }^{6} \mathrm{C}$ wm Taf University Health Board, Llantrisant, UK; ${ }^{7}$ Norfolk and Norwich University Hospital, Norwich, UK

10.1136/gutjnl-2019-BSGAbstracts.478
Introduction Validated competency assessment tools and the data supporting milestone development during gastroscopy training are lacking. We aimed to assess the validity of a novel formative direct observation of procedural skills (DOPS) assessment tool in gastroscopy, and study competency development using DOPS.

Methods This was a prospective multicentre analysis of formative gastroscopy DOPS assessments. Internal structure validity was tested using exploratory factor analysis and reliability estimated using generalisability theory. Item and global DOPS scores were stratified by lifetime procedure count to define learning curves, using a threshold determined from receiver operator characteristics (ROC) analysis. Multivariable binary logistic regression analysis was performed to identify independent predictors of DOPS competence. 


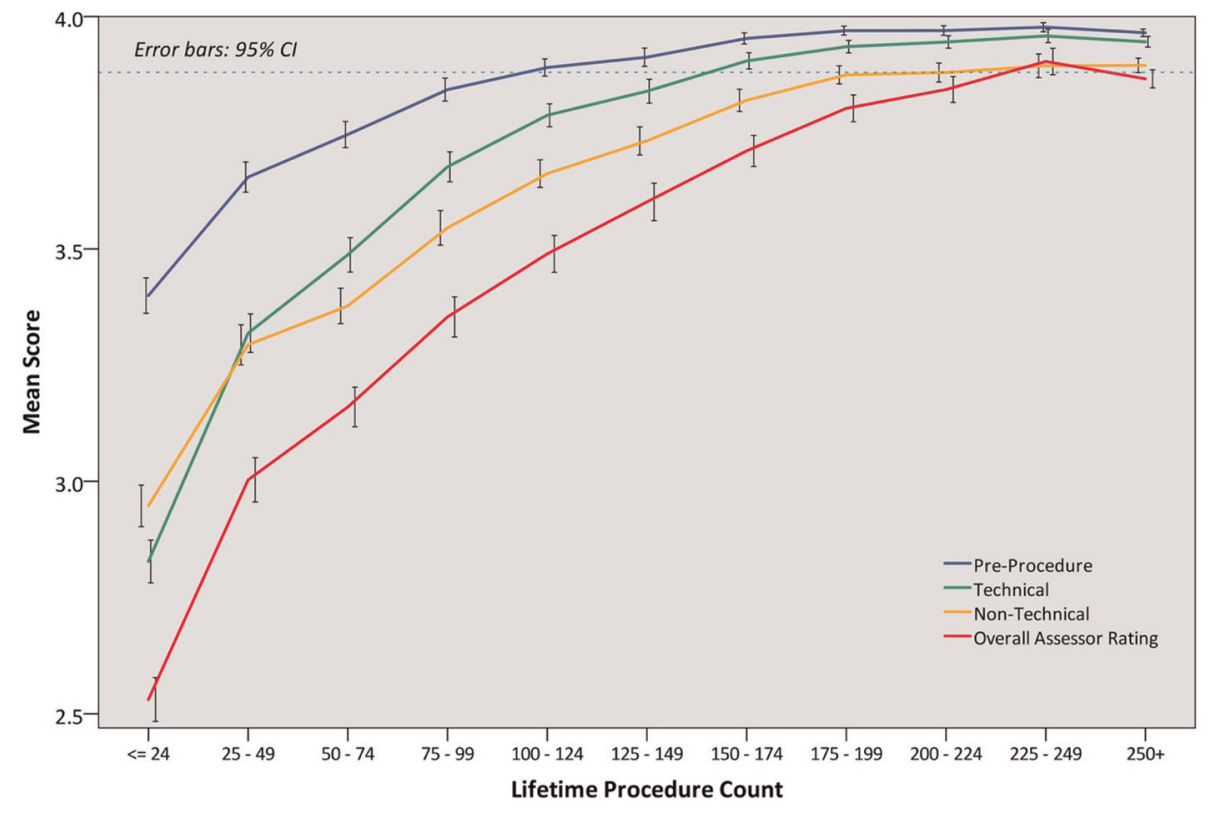

Abstract PWE-107 Figure 1 Learning curves in gastroscopy as assessed by the constructs identified in factor analysis: a) Pre-procedure, b) Technical (covering Insertion \& Withdrawal and Visualisation domains), c) Non-technical (covering Management, Post-Procedure and ENTS domains).

Results 10086 DOPS were submitted for 987 trainees from 275 UK training centres. Exploratory factor analysis identified three distinct item groupings: representing 'pre-procedure', 'technical' and 'post-procedure non-technical' skills. From generalisability analyses, sources of variance in overall DOPS scores included: trainee ability (31\%), assessor stringency $(8 \%)$, assessor subjectivity (18\%), and trainee case-to-case variation (43\%). The combination of 3 assessments from 3 assessors was sufficient to achieve the acceptable reliability threshold of 0.70 . On ROC analysis, a mean score of 3.9 provided optimal sensitivity and specificity for determining competency. This threshold was attained in the order of: 'preprocedure' (10-24 procedures), 'technical' (15-74 procedures), 'post-procedure non-technical' skills (20-24 procedures), and overall competency (22-49 procedures) [Abstract PWE107 Figure 1]. Higher lifetime procedure count $(\mathrm{P}<0.001)$, DOPS count $(\mathrm{P}<0.001)$, trainee specialty $(\mathrm{P}=0.028)$ and assessor specialty $(\mathrm{P}=0.002)$, trainee seniority $(\mathrm{P}=0.011)$ and lower case difficulty $(\mathrm{P}<0.001)$ were multivariable predictors of DOPS competence.

Conclusion This study establishes milestones for competency acquisition during gastroscopy training and provides novel validity and reliability evidence to support gastroscopy DOPS as a competency assessment tool.

\section{PWE-108 THE FLEXIBLE SIGMOIDOSCOPY DOPS ASSESSMENT TOOL: EVIDENCE OF VALIDITY AND COMPETENCY DEVELOPMENT DURING TRAINING}

${ }^{1}$ Keith Siau*, ${ }^{2}$ Prof James Crossley, 1,3Paul Dunckley, ${ }^{1,4}$ Gavin Johnson, ${ }^{5}$ Adam Haycock, ${ }^{1,3}$ John Anderson, ${ }^{6}$ Marietta lacucci, ${ }^{1,7}$ Mark Feeney. 'Joint Advisory Group on Gastrointestinal Endoscopy, London; ${ }^{2}$ University of Sheffield, Sheffield; ${ }^{3}$ Gloucestershire Hospitals NHSFT, Gloucestershire; ${ }^{4}$ University College London Hospitals NHSFT, London; ${ }^{5}$ St Mark's Hospital, London; ${ }^{6}$ University of Birmingham, Birmingham; ${ }^{7}$ Torbay and South Devon NHS Foundation Trust, Torquay
Introduction Data supporting milestone development during flexible sigmoidoscopy (FS) training are lacking. We aimed to present validity evidence for our formative direct observation of procedural skills (DOPS) assessment in FS, and use DOPS to establish competency benchmarks and define learning curves for a national training cohort.

Methods This prospective UK-wide (211 centres) study included all FS formative DOPS assessments submitted to the national e-portfolio. Reliability was estimated from generalisability theory analysis. Item and global DOPS scores were correlated with lifetime procedure count to study learning curves, with competency benchmarks defined using contrasting groups analysis. Multivariable binary logistic regression was performed to identify independent predictors of DOPS competence.

Results 3616 DOPS submitted for 468 trainees were included for analysis. From generalisability analysis, sources of overall competency score variance included: trainee ability (27\%), assessor stringency (15\%), assessor subjectivity attributable to the trainee (18\%) and case-to-case variation (40\%), which enabled the modelling of reliability estimates. The competency benchmark (mean DOPS score: 3.84) was achieved after 15-74 procedures. Across the cohort, competency development occurred in the order of: pre-procedural (5-4), non-technical (7-49), technical (12-74) and post-procedural (17-99) skills (Abstract PWE108 Figure 1). Lifetime procedural count $(\mathrm{P}<0.001)$, case difficulty $(\mathrm{P}<0.001)$, and lifetime formative DOPS count $(\mathrm{P}=0.001)$ were independently associated with DOPS competence, but not trainee or assessor specialty.

Conclusion Sigmoidoscopy DOPS can provide valid and reliable assessments of competency during training and can be used to chart competency development. Contrary to earlier studies based on destination-orientated endpoints, overall competency in sigmoidoscopy was attained after 150 lifetime procedures. 